\title{
Galilei Invariant Molecular Dynamics
}

\author{
By \\ Christian D. JÄKEL * Günther HÖRMANN **
}

\begin{abstract}
We construct a model for a chemical reaction in the Heisenberg representation. The time evolution exists in the thermodynamic limit, independent of the particle density and the initial state. In mathematical terms, we establish a $\mathrm{C}^{*}$-dynamical system. Thus we can benefit from general results in algebraic quantum statistical mechanics, showing, for example, that equilibrium states exist. Galilei invariance of our nonrelativistic model is demonstrated by defining it directly on the Galilean space-time manifold, without reference to any coordinate system. PACS 05.30.Fk, 03.70. + k. 11.10.Cd, 11.15.Tk
\end{abstract}

\section{§1. Introduction}

Many of the properties typically attributed to relativistic field theories are actually a common feature of every theory with a zero mean-particle density and translation-invariant Hamiltonian [1]. Galilei invariant quantum field theories provide a natural candidate for a comparison with relativistic models. Especially the Galilei invariant Lee model has attracted much attention [2-4]. Most of the work in nonrelativistic field theory was based on the Hamiltonian approach and constructed in the vacuum representation. More recently, thermo field theories aimed at the construction of systems in an equilibrium state at finite temperature.

The usual procedure is to define a model in some kind of "box", then let the box go to infinity and show that the thermodynamic limit is independent of the boundary conditions involved in the definition of the system. While one could argue that a box is in agreement with the experimental situation, one should keep in mind that for macroscopic bodies it is not possible to isolate the energy levels completely - their widths are on the order of (macroscopic time $)^{-1}$, which

Communicated by H. Araki, November 14, 1994.

1991 Mathematics Subject Classifications: 46L40, 20C35, 81V45

*II. Institut für Theoretische Physik, Universität Hamburg, D-22761 Hamburg, Germany

**Institut fur Mathematik, Universität Wien, A-1090 Wien, Austria 
is much larger than their spacing. So in order to avoid mathematical curiosities comming from periodic boundary conditions and a discrete spectrum it is better to idealize macroscopic bodies as being infinite and having continuous energy spectra, which comes closer to reality than does the fiction of a discrete spectrum. But for an infinite sytem, even for a finite particle density, one has to face the possibility of unitary inequivalent representations. In fact, if the algebra of observables changes globally as time passes, as is expected for almost any initial state, then a representation may change at any moment into an inequivalent representation, and it is not possible to represent the time-evolution with a group of unitary transformations within the representation (cf. [10]). Thus even for the free time evolution $\tau^{0}$ one can not expect that a formal expression like

$$
\mathbb{H}_{0}=\frac{1}{2 m} \int \mathrm{d}^{3} x a^{*}(\nabla x) a(\nabla x)
$$

will exist as a selfadjoint operator acting on a certain Hilbert space for an arbitrary initial state. Nevertheless, at least for fermions, the free time evolution exists as a strongly continuous automorphism $t \rightarrow \tau_{t}^{0}$ acting on a $\mathrm{C}^{*}$-algebra, which describes the possibilities of testing the system experimentally. Only recently it was demonstrated by Narnhofer and Thirring $[5,6]$ that pair interaction can be added to the free time evolution. There one has to face a problem known as "stability of matter", which roughly speaking tells us that independent of the initial conditions the system does not heat up and collapse.

In this article we demonstrate that even a chemical reaction can be added to a system of fermions with pairinteraction. In $\S 2$ we define the appropriate $\mathrm{C}^{*}$-algebra of observables $\mathscr{A}$ for our model. We deal with three different species of fermions. Different methods for the construction of automorphisms of the algebra of observables $\mathscr{A}$ are discussed, and some remarks on the superselection structure are added. In $\S 3$ we present our " $\mathrm{C}^{*}$-dynamical" model for a chemical reaction, which was inspired by the Galilei invariant Lee model cited above. As in the Narnhofer-Thirring model, the existence of the time evolution is achieved by cutting off high relative momenta, thereby introducing a slightly non-local character of the interaction. The time development is well defined for arbitrary initial states, and in principle one could tackle problems of nonequilibrium situations where the state changes globally with time and the time evolution $\tau$ can not be unitarily implemented in the GNS-representation corresponding to a state at a fixed time. But work in this direction seems difficult and much remains to be done. Mixing properties will be discussed elsewhere.

The advantage of formulating our model as a " $\mathrm{C}^{*}$-dynamical system" is that the algebraic formulation of quantum statistical mechanics [7-10] and quantum 
ergodic theory [11-17] become applicable. We can only mention a few basic results here, for further details and properties the reader is refered to the literature cited. In $\$ 5$ we answer the question whether or not our model has equilibrium states. The positive answer is based on a result by Powers and Sakai [18]. By definition, ground and equilibrium states are time-invariant, so in the corresponding GNS representation $\pi_{\beta}$ the time evolution $\tau$ can be represented by a Hamilton operator $\mathbb{H}_{\beta}$,

$$
\pi_{\beta}\left(\tau_{t}(a)\right)=e^{\imath t \mathbf{H}_{\beta}} \pi_{\beta}(a) e^{-\imath t \mathbf{H}_{\beta}}, \quad a \in \mathscr{A}
$$

where the representations $\pi_{\beta}$ are labeled by the inverse temperature $\beta=1 / k T$ of the equilibrium state ${ }^{1}$. This establishes the connection with the standard construction starting from a system in a box mentioned above. The other vector states now represent local excitations of the equilibrium state and differ only locally, and thus do not change in time, from a global point of view. We will also point out that equilibrium states spontaneously break Galilei invariance and that even a local perturbation changes the number of parameters labelling different equilibrium states.

While our model might well serve as an effective theory for a chemical reaction in studying thermodynamical properties, we can not expect phenomenological applications. Although we consider molecules as the elementary objects, we have resisted, in order to keep the notational amount acceptable, to include the two-particle interactions responsible for the formation of molecules. It is not hard to check that our model can be combined with the two-particle interactions recently presented by Narnhofer and Thirring $[5,6]$ as well as similar twoparticle interactions between different species.

In quantum mechanics space and time coordinates do not refer to an individual particle but refer to preparation and registration apparatuses. The 'classical' space-time structure is merely encoded in the net of local algebras. This aspect is worked out in an appendix, where the algebra of observables is equipped with the fiber-bundle structure induced by the structure of spacetime. The symmetry group of 'nonrelativistic' space-time is of course the Galilei group $[19,20]$. In fact, the Galilei group provides the transition functions between different charts of the space-time manifold ${ }^{2}$. In $\S 4$ we show that the time-evolution is Galilei invariant, i.e., it can be defined chart independently on the new bundle. While this might look extraordinary for the first sight, we

\footnotetext{
${ }^{1} k$ denotes the Boltzmann constant.

${ }^{2}$ The representation theory of the Galilei group on Hilbert spaces is well established [19, 20]. Recently there has been some interest in more abstract aspects of this group [21-26]. Nevertheless the Banach space representations we construct in the appendix seem to have no counterpart in literature.
} 
feel that it is the natural, coordinate free formulation of Galilei invariance. That we are able to define a time-evolution in a coordinate-free way makes it evident that only the spatial relationship between the preparation and registration apparatuses are relevant.

\section{§2. The Algebra of Observables}

We want to describe molecular dynamics involving chemical reactions such as the dissociation of large molecules into two different smaller parts, for example a four atomic molecule which dissociates into two simple molecules:
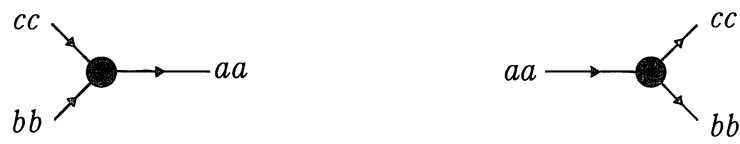

Since the difference between various isotopes are of no relevance for us we assume that all the particles are fermions. The relevant $\mathrm{C}^{*}$-algebra is then the 3 -fold tensor product of the usual CAR or Fermi algebra $\mathscr{A}_{F}$ for one species of fermions. Uniqueness of this tensor product is guaranteed by the fact the Fermi algebra $\mathscr{A}_{F}$ is nuclear [8].

Definition. Let $\mathscr{A}$ denote the unital $C^{*}$-algebra generated by annihilation and creation operators $a(f), a^{*}(f), b(f), b^{*}(f), c(f)$ and $c^{*}(f)$, where $f \in$ $L^{2}\left(\mathbb{R}^{3}\right)$. These operators correspond to three different particle species with masses $m_{a}=m_{b}+m_{c}$ and obey anticommutation relations

$$
\left\{a(f), a^{*}(g)\right\}=(f \mid g) \mathbb{1}, \quad\{a(f), a(g)\}=0,
$$

and similarly for $b$ and $c$. Operators corresponding to particles of different species commute. Thus $\mathscr{A}=\mathscr{A}_{a} \otimes \mathscr{A}_{b} \otimes \mathscr{A}_{c} ; \mathscr{A}_{x} \cong \mathscr{A}_{F}, x=a, b, c$.

Note that we consider $\mathscr{A}$ as an abstract algebra - representations of $\mathscr{A}$ in certain Hilbert spaces will be state dependent and discussed in $\S 5$. If we are interested in obtaining a Galilei invariant quantum field theory exhibiting production processes, we have to include particles with mass values chosen in agreement with the mass conservation law. This was done in the definition of $\mathscr{A}$. As noticed by Bargmann [19], the mass operator $\mathbf{M}$, appearing as an element of the center of the extended Galilei group Lie algebra, gives rise to a superselection rule. In fact, as long as we have no chemical reactions the superselection sectors are labelled by three charges, corresponding to the three different total masses $\mathbb{M}=\mathbf{M}_{a}+\mathbf{M}_{b}+\mathbf{M}_{c}$. The corresponding equilibrium states are labeled 
by three distinct chemical potentials. A chemical interaction will change the superselection structure. Besides the total mass only one more parameter will survive. If the particles are charged, then this parameter turns out to be the total charge.

We list three different procedures for the construction of automorphisms $\gamma$ of $\mathscr{A}$. Their common feature is that $\gamma(a(f)), \gamma(b(f))$ and $\gamma(c(f))$ are specified on a total set $\mathscr{T}$ of functions $f \in \mathscr{T} \subset L^{2}\left(\mathbf{R}^{3}\right)$. Then this definition is extended to the whole generating system of $\mathscr{A}$ by setting $\gamma(\mathbf{1}):=\mathbf{1}$ and $\gamma\left(a^{*}(f)\right):=(\gamma(a(f)))^{*}$ and similarly for $b$ and $c$. If now $\gamma$ respects the defining relations among the generators, it can be extended to a ${ }^{*}$-isomorphism of $\mathscr{A}$ (again denoted by $\gamma$ ), because of the uniqueness of the CAR-algebra [8] and the involved tensor products.

(i) Coherent states

$$
f_{z}(x):=\pi^{-\frac{3}{4}} \mathrm{e}^{-\frac{1}{2}(x-q)^{2}+\imath p(x-q)} \in L^{2}\left(\mathbf{R}^{3}, \mathrm{~d}^{3} x\right), \quad z=(q, p) \in T^{*}\left(\mathbf{R}^{3}\right)
$$

explicitly relate to the classical picture. We may think of $a_{z}:=a\left(f_{z}\right)$ as an operator destroying a particle centered at $z=(q, p)$ in phase space. This corresponence between the classical and the quantum picture allows us to lift group representations from ordinary phase space $T^{*}\left(\mathbf{R}^{3}\right)$ to groups of automorphisms of $\mathscr{A}$. For example, the kinematical automorphisms

$$
\gamma_{g}^{K i n}(t)\left(a_{z}\right):=\mathrm{e}^{\imath m a \lambda+\imath m a \frac{v^{2}}{2}(t-s)+\imath m a v \cdot R a} a_{\bar{z}}, \quad \bar{z}=\left(R q+v(t-s)+l, R p+m_{a} v\right),
$$

$g=(\lambda, s, l, v, R)$, define a representation of the extended Galilei group $G^{1}$ in Aut $(\mathscr{A})$. (The phase-factor was chosen in agreement with the cocycle relation of the Galilei group extension.)

(ii) The standard procedure to lift unitary representations of groups from the quantum mechanical one-particle space to quasifree automorphisms of the algebra was introduced by Bogoliubov [8]. Since the kinematical automorphisms are quasifree we could as well use representations of the Galilei group in coordinate-space $[20]$ :

$$
f(x) \rightarrow \mathrm{e}^{\imath m \lambda-i m \frac{v^{2}}{2}(t-s)+\imath m v \cdot(x-l)} f\left(R^{-1}(x-l-v(t-s))\right), \quad f \in L^{2}\left(\mathbf{R}^{3}\right)
$$

for a construction of these automorphisms. The free time evolution $\tau_{t}^{0}(a(f)):=$ $a\left(\mathrm{e}^{-\imath \frac{\Delta}{2 m a} t} f\right)$ provides another example. 
(iii) Each hermitian element $h=h^{*}$ of $\mathscr{A}$ defines a so-called inner automorphism $\gamma^{h}$ :

$$
\gamma_{t}^{h}:=\mathrm{e}^{i t h} a \mathrm{e}^{-i t h}, \quad a \in \mathscr{A}, \quad t \in \mathbb{R}
$$

Since $\mathscr{A}$ is a Banach space, we are allowed to consider limits. The limit $\gamma_{t}:=$ $\lim _{n \rightarrow \infty} \gamma_{t}^{h_{n}}$ might exist even when $\left\{h_{n}\right\}_{n \in \mathbf{N}}$ does not converge in $\mathscr{A}$. Automorphisms which are not inner are called outer automorphisms.

The Lie algebra of the extended Galilei group (see Appendix) tells us that gauge transformations have to commute with time translations. We might therefore think of the gauge group as representing inner symmetries. In addition we expect gauge-dependent quantities to be unobservable. Nevertheless a chemical reaction, corresponding to an operator of the form $a^{*} b c$, has to be observable. This suggests the following choice for the action of gauge transformations on $\mathscr{A}$.

Definition. The action of the gauge group $U(1)$ as a group of

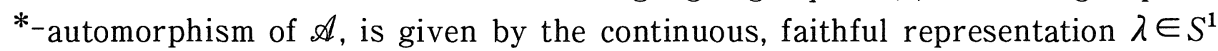
$\rightarrow \gamma_{\lambda}$, defined by algebraic extension of their action on the generating elements of $\mathscr{A}$,

$$
a(f) \otimes_{b}(f) \otimes_{c}(f) \rightarrow \mathrm{e}^{i \lambda\left(m_{a}+m_{b}+m_{c}\right)} a(f) \otimes_{b}(f) \otimes_{c}(f)
$$

and topological closure ${ }^{3}$. The fixed-point algebra under the action of $U(1)$ is called the algebra of observables.

\section{§3. A Thermodynamical System with Chemical Reactions}

While the Narnhofer-Thirring model is concerned with pair interactions, of similar molecules with (regularised) Van der Waal's interaction for example, the present effective interaction only includes vertex functions that model the restructuring of the electrons once the molecules have got so close to one another that their electron clouds overlap. In fact, our model describes only a certain chemical reaction of diatomic molecules. The existence of diatomic molecules is assumed and the forces responsible for the formation of molecules are not dealt with.

We construct the interacting time-evolution in three steps, as proposed by Guenin [27]:

\footnotetext{
${ }^{3}$ That $\gamma_{\lambda}$ is well defined can be easily checked by computing the new anticommutationrelations.
} 
(i) We "cut-off" the interaction in such a way that $v_{n}$ exists as an element in $\mathscr{A}$, thereby destroying Galilei invariance.

(ii) We define inner automorphisms $\tau^{n}$ corresponding to the "cut-off" interaction $v_{n}$.

(iii) We construct the interacting time-evolution as a limit of inner automorphisms by releasing the cut-offs and thereby restoring Galilei invariance.

Diatomic molecules are idealised as two atoms at a fixed distance $\hat{q}$ and with equal momentum. The spatial orientation of the molecular axis should be of secondary importance and is therefore neglected.

Definition. Let $\tau_{s}:=s-\lim _{n \rightarrow \infty} \tau_{s}^{n}$, where $s-\lim$ denotes the strong (i.e., pointwise) limit in $\mathscr{B}(\mathscr{A})$ - the Banach space of bounded operators acting on $\mathscr{A}$ - of the following automorphisms $\tau_{s}^{n}$.

$\tau_{s}^{n}(a):=\tau_{s}^{0}(a)+\sum_{k=1}^{\infty} \frac{i^{k}}{k !} \int_{[0, s]^{k}} \mathrm{~d}\left(t_{1} \ldots t_{k}\right)\left[\tau_{t_{k}}^{0}\left(v_{n}\right),\left[\ldots,\left[\tau_{t_{1}}^{0}\left(v_{n}\right), \tau_{s}^{0}(a)\right] \ldots\right]\right], \quad a \in \mathscr{A}$,

where $\mathrm{d}\left(t_{1} \ldots t_{k}\right)$ denotes the time ordered integral (however over $[0, s]^{k}$ and hence divided by $k$ !) and

$$
\begin{aligned}
& v_{n}=\int_{\left|q_{1}\right|,\left|p_{n}\right| \leq n} \prod_{i=1}^{3} \mathrm{~d}^{3} q_{\imath} \mathrm{d}^{3} p_{\imath} \int \mathrm{d} O V\left(\left|q_{1}-q_{2}\right|,\left|q_{1}-q_{3}\right|,\left|\frac{p_{1}}{m_{a}}-\frac{p_{2}}{m_{b}}\right|,\left|\frac{p_{1}}{m_{a}}-\frac{p_{3}}{m_{c}}\right|, \vartheta_{q}, \vartheta_{p}\right)
\end{aligned}
$$

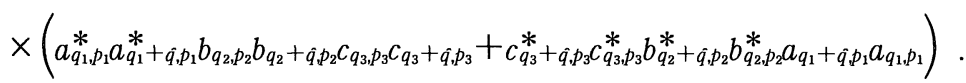

$\vartheta_{q}$ denotes the angle between $\left(q_{1}-q_{2}\right)$ and $\left(q_{1}-q_{3}\right)$ and $\vartheta_{p}$ between $\left(\frac{p_{1}}{m_{a}}-\frac{p_{2}}{m_{b}}\right)$ and $\left(\frac{p_{1}}{m_{a}}-\frac{p_{3}}{m_{c}}\right)$ and $\mathrm{d} O$ denotes the surface integral over the sphere $|\hat{q}|=$ const. The vertex $V\left(|r|,\left|r^{\prime}\right|,|k|,\left|k^{\prime}\right|, \vartheta_{q}, \vartheta_{p}\right)$ is assumed to be in $L^{1}\left(\mathbb{R}^{12}, \mathrm{~d}^{3} r \mathrm{~d}^{3} r^{\prime} \mathrm{d}^{3} k \mathrm{~d}^{3} k^{\prime}\right)$ $\cap C\left(\mathbb{R}^{12}\right)$, thus all Bochner-integrals exist.

The interaction is constructed so that there are two conservation laws: the number of $a$-particles plus the number of $b$-particles is conserved, and the number of $b$ particles minus the number of $c^{-}$-particles. The vertex function $V$ should be localised in a region of order of the atomic distances $\hat{q}$. The momentum de pendence of the vertex function is unknown, but we have only required integrability and this seems to be a decent assumption. We have made no restrictions on the angular dependence of the position and the momentum distributions since 
$V$ will certainly depend on $\vartheta_{q}$ and $\vartheta_{p}$. Furthermore, our six-particle vertex will suppress dissociation with very high relative momenta of the molecules. However, there will be no limit on the momentum cut-off and one expect that for stable systems where high momenta do not occur in a reasonable subset of states, our interaction gives a physically acceptable description.

One can interpret the presented model alternatively as one involving particle creation and annihilation. In any case, it exists strictly on the $\mathrm{C}^{*}$-algebraic level, without any renormalization or restriction to any representation. Before we show that the limits involved in the definition of the time evolution exist, we mention that the interaction is stable for both signs of the coupling constant. It was argued in [14] that this is necessary, if the interaction defines a strongly continuous automorphism. Furthermore it automatically ensures a reasonable thermodynamical behaviour of the system by guaranteeing that the energy is an extensive quantity, which is a basic assumption in phenomenological thermodynamics.

Lemma 3.1. The interaction is stable ${ }^{4}$, i.e., there exists a positive real number $C \in \mathbf{R}^{+}$, such that

$$
h_{n}:=\left(h_{a}^{\circ}\right)_{n}+\left(h_{b}^{\circ}\right)_{n}+\left(h_{c}^{\circ}\right)_{n}+v_{n}>-C\left(\left(N_{a}\right)_{n}+\left(N_{b}\right)_{n}\right) \text {, }
$$

where $\left(h_{a}^{\circ}\right)_{n}=\frac{1}{2 m_{a}} \int_{|q|,|p| \leq n} \mathrm{~d}^{3} q \mathrm{~d}^{3} p \quad a^{*}\left(\nabla f_{q, p}\right) a\left(f_{q, p}\right) \geq 0$,

and $\left(N_{a}\right)_{n}=\int_{|q|,|p| \leq n} \mathrm{~d}^{3} q \mathrm{~d}^{3} p \quad a_{q, p}^{*} a_{q, p} \geq 0$, and similiar for $b, c$.

Proof. We first show that $x+x^{*} \leq \sqrt{2}\left(x^{*} x+x x^{*}\right)^{1 / 2}$. From $\left(x-x^{*}\right)^{*}(x-$ $\left.x^{*}\right) \geq 0$ we infer

$$
x^{*} x+x x^{*} \geq x^{2}+\left(x^{*}\right)^{2} .
$$

Thus $2\left(x^{*} x+x x^{*}\right) \geq\left(x+x^{*}\right)^{2}$. We simplify the notation,

$$
z_{\imath}=\left(q_{i}, p_{i}\right), \quad \hat{z_{i}}=\left(q_{i}+\hat{q}, p_{i}\right)
$$

and recall that operators corresponding to different particles commute. Thus

$$
-v_{n}=-\int_{\left|q_{1}\right|,\left|p_{1}\right| \leq n} \mathrm{~d}^{6} z_{1} \mathrm{~d}^{6} z_{2} \mathrm{~d}^{6} z_{3} \int_{|q|=\text { const. }} \mathrm{d} O \quad V(\ldots) \quad\left(a_{z_{1}}^{*} a_{z_{1}}^{*} b_{z_{2}} b_{z_{2}} c_{z_{3}} c_{z_{3}}+\text { herm.conj. }\right)
$$

\footnotetext{
${ }^{4}$ This result evolved from discussions with E. Lieb, D. Buchholz, H. Narnhofer and R. Verch.
} 


$$
\begin{gathered}
\leq \sqrt{2} \int_{\left|q_{1}\right|,\left|p_{1}\right| \leq n} \mathrm{~d}^{6} z_{1} \mathrm{~d}^{6} z_{2} \mathrm{~d}^{6} z_{3} \int \mathrm{d} O\left|V\left(\left|q_{1}-q_{2}\right|,\left|q_{1}-q_{3}\right|,\left|\frac{p_{1}}{m_{a}}-\frac{p_{2}}{m_{b}}\right|,\left|\frac{p_{1}}{m_{a}}-\frac{p_{3}}{m_{c}}\right|, \vartheta_{q}, \vartheta_{p}\right)\right| \\
\quad \times\left(c_{z_{3}}^{*} z_{z_{3}}^{*} b_{z_{2}}^{*} b_{z_{2}}^{*} a_{\bar{z}_{1}} a_{z_{1}} a_{z_{1}}^{*} a_{z_{1}}^{*} b_{z_{2}} b_{z_{2}} c_{z_{3}} c_{z_{3}}+a_{z_{1}}^{*} a_{z_{1}}^{*} b_{z_{2}} b_{z_{2}} c_{z_{3}} c_{z_{3}} c_{z_{3}}^{*} c_{z_{3}}^{*} b_{z_{2}}^{*} b_{z_{2}}^{*} a_{\bar{z}_{1}} a_{z_{1}}\right)^{1 / 2}
\end{gathered}
$$

Next we apply the operator inequality

$$
a b b^{*} a^{*} \leq a a^{*}\|b\|\|b *\|
$$

and use the operator monotony of the square root. Once more it is crucial that operators corresponding to different particles commute. We find

$$
\begin{aligned}
& -v_{n} \leq \sqrt{2} \int_{\left|q_{1}\right|, p_{1} \mid \leq n} \mathrm{~d}^{6} z_{1} \mathrm{~d}^{6} z_{2} \mathrm{~d}^{6} z_{3} \int \mathrm{d} O\left|V\left(\left|q_{1}-q_{2}\right|,\left|q_{1}-q_{3}\right|, \ldots, \vartheta_{q}, \vartheta_{p}\right)\right|\left(b_{z_{2}}^{*} b_{z_{2}}+a_{z_{1}}^{*} a_{z_{1}}\right)^{1 / 2} \\
& \leq \sqrt{2} 4 \pi|\hat{q}|^{2} \sup _{q_{2}, p_{2}}\left(\int_{\left|q_{1}\right|,\left|p_{1}\right| \leq n} \mathrm{~d}^{6} z_{1} \mathrm{~d}^{6} z_{3}\left|V\left(\left|q_{1}-q_{2}\right|,\left|q_{1}-q_{3}\right|, \ldots, \vartheta_{q}, \vartheta_{p}\right)\right|\right) \int_{\left|q_{2}\right|,\left|p_{2}\right| \leq n} \mathrm{~d}^{6} z_{2} b_{z_{2}}^{*} b_{z_{2}} \\
& +\sqrt{2} 4 \pi|\hat{q}|^{2} \sup _{q_{1}, p_{1}}\left(\int_{\left|q_{1}\right|,\left|p_{1}\right| \leq n} \mathrm{~d}^{6} z_{2} \mathrm{~d}^{6} z_{3}\left|V\left(\left|q_{1}-q_{2}\right|,\left|q_{1}-q_{3}\right|, \ldots, \vartheta_{q}, \vartheta_{p}\right)\right|\right) \int_{\left|q_{1}\right|,\left|p_{1}\right| \leq n} \mathrm{~d}^{6} z_{1} a_{z_{1}}^{*} a_{z_{1}} \\
& \leq \sqrt{2} 4 \pi|\hat{q}|^{2} m_{a}^{6}\|V\|_{1}\left(\int_{\left|q_{2}\right|,\left|p_{2}\right| \leq n} \mathrm{~d}^{6} z_{2} b_{z_{2}}^{*} b_{z_{2}}+\int_{\left|q_{1}\right|,\left|p_{1}\right| \leq n} \mathrm{~d}^{6} z_{1} a_{z_{1}}^{*} a_{z_{1}}\right)
\end{aligned}
$$

In the last two inequalities we used the fact that $P_{a}=a_{z}^{*} a_{z}$ and $P_{b}=b_{z}^{*} b_{z}$ are commuting projection operators and therefore $\left(P_{a}+P_{b}\right)^{1 / 2} \leq P_{a}+P_{b}$.

Thus $-v_{n}<\sqrt{2} 4 \pi||^{2} m_{a}^{6}\|V\|_{1}\left(\left(N_{a}\right)_{n}+\left(N_{b}\right)_{n}\right)$, and similar $-v_{n}<\sqrt{2} 4 \pi|q|^{2} m_{a}^{6}\|V\|_{1}\left(\left(N_{a}\right)_{n}+\left(N_{c}\right)_{n}\right)$.

Stability for the opposite sign follows by the same line of arguments. The inequality (11) gives a bound on the chemical reaction rate, which depends on two different processes. First, the heavy molecules can dissipate by their own, without a partner for a reaction. Thus their number $N_{a}$ has to be included in all bounds on the reaction rate. Secondly, the formation of the larger molecule of type $a a$ is limited by the more seldom reaction partner.

Theorem 3.2. $\tau$ is well defined (as a strongly continuous one-parameter group of automorphism of $\mathscr{A}$.)

Proof. We show that the perturbation formula (9) for $\tau_{t}^{n}\left(a_{z^{\prime}}\right)$ converges for all $z \in T^{*}\left(\mathbf{R}^{3}\right)$ as $n \rightarrow \infty$. We proceed in three steps. 
(i) The number of terms does not increase too fast. The strategy is to rewrite commutators in terms of anticommutators. The first order in the coupling constant is

$$
\begin{aligned}
\int_{0}^{t} \mathrm{~d} t_{1} \int_{\left|q_{s}\right|||_{p} \mid \leq n} \mathrm{~d}^{6} z_{1} \mathrm{~d}^{6} z_{2} \mathrm{~d}^{6} z_{3} \int_{|q|=\text { const. }} \mathrm{d} O & V\left(\left|q_{1}-q_{2}\right|,\left|q_{1}-q_{3}\right|,\left|\frac{p_{1}}{m_{a}}-\frac{p_{2}}{m_{b}}\right|,\left|\frac{p_{1}}{m_{a}}-\frac{p_{3}}{m_{c}}\right|, \vartheta_{q}, \vartheta_{p}\right) \\
\times & \left.\times \tau_{t_{1}}^{\circ}\left(a_{z_{1}}^{*} a_{z_{1}}^{*} b_{z_{2}} b_{z_{2}} c_{z_{3}} c_{z_{3}}+c_{z_{3}}^{*} c_{z_{3}}^{*} b_{z_{2}}^{*} b_{z_{z}}^{*} a_{z_{1}}^{*} a_{\tilde{z}_{1}}\right), \tau_{t}^{\circ}\left(a_{z^{\prime}}\right)\right]
\end{aligned}
$$

As one can see from this expression, every new order in the coupling constant introduces two products of six creation and annihlitation operators. Given a fixed creation operator (resp. annihilation operator) only one of these two terms has a nonvanishing commutator. For example, in the first order we find:

$$
\begin{aligned}
{\left[\tau_{t_{1}}^{\circ}\left(a_{z_{1}}^{*} a_{z_{1}}^{*}\right), \tau_{t}^{\circ}\left(a_{z^{\prime}}\right)\right] \tau_{t_{1}}^{\circ}\left(b_{z_{2}} b_{\tilde{z}_{2}} c_{z_{3}} c_{z_{3}}\right)=} & \left\{\tau_{t_{1}}^{\circ}\left(a_{z_{1}}^{*}\right), \tau_{t}^{\circ}\left(a_{z^{\prime}}\right)\right\} \tau_{t_{1}}^{\circ}\left(a_{z_{1}}^{*} b_{z_{2}} b_{z_{2}} c_{z_{3}} c_{z_{3}}\right) \\
& +\left\{\tau_{t_{1}}^{\circ}\left(a_{z_{1}}^{*}\right), \tau_{t}^{\circ}\left(a_{z^{\prime}}\right)\right\} \tau_{t_{1}}^{\circ}\left(a_{z_{1}}^{*} b_{z_{2}} b_{\hat{z}_{2}} c_{z_{3}} c_{\tilde{z}_{3}}\right)
\end{aligned}
$$

Thus we are left with two products of five $(=4+1)$ creation and annihilation operators. If we expand the next commutator into anticommutators each of the $4+1$ creation and annihilation operators has two non vanishing anticommutators with the interaction. Therefore in second order we have to compute bounds for $2 \cdot(4+1) \cdot 2$ terms of lengh $2 \cdot 4+1$. By the same line of arguments the number of nonvanishing terms in third order is given by $2 \cdot(4+1) \cdot 2 \cdot(8+$ 1) $\cdot 2$. For arbitrary order $k$ we find $2 \cdot(4+1) \cdot 2 \cdot(2 \cdot 4+1) \cdot \ldots \cdot(k \cdot 4+1) \cdot 2$ and taking the factor $1 / k$ ! in equation (9) into account we arrive at

$$
\frac{2 \cdot(4+1) \cdot 2 \cdot(2 \cdot 4+1) \cdot \ldots \cdot 2 \cdot(k \cdot 4+1) \cdot 2}{1 \cdot 2 \cdot 3 \cdot \ldots \cdot k}=2^{k+1} \cdot(4+1) \cdot\left(4+\frac{1}{2}\right) \cdot \ldots \cdot\left(4+\frac{1}{k}\right) \leq 2 \cdot(2 \cdot 5)^{k}
$$

(ii) All $\tau_{t}^{n}\left(a_{z^{\prime}}\right)$ can be bounded uniformly in $n \in \mathbb{N}$ and $z^{\prime} \in \mathbb{R}^{6}$. First we note that

$$
\left\|\tau_{t}^{n}\left(a_{z^{\prime}}\right)\right\| \leq\left\|\tau_{t}^{\circ}\left(a_{z^{\prime}}\right)\right\|+\sum_{k=1}^{\infty}\left\|\lim _{m \rightarrow \infty} \frac{1}{k !} \int_{[0, t]^{k}} \mathrm{~d}\left(t_{1} \ldots t_{k}\right)\left[\tau_{t_{k}}^{\circ}\left(v_{m}\right),\left[\ldots,\left[\tau_{t_{1}}^{\circ}\left(v_{m}\right), \tau_{t}^{\circ}\left(a_{z^{\prime}}\right)\right] \ldots\right]\right]\right\|
$$

for all $n \in \mathbb{N}$. The next step is to use the fact that the spreading of free coherent wave functions $f_{z^{\prime}}$ does not depend on the footpoint $z^{\prime} \in T^{*}\left(\mathbb{R}^{3}\right)$ : 


$$
\int \mathrm{d}^{6} z\left\|\left\{\tau_{t}^{\circ}\left(a_{z}^{*}\right), \tau_{t^{\prime}}^{\circ}\left(a_{z^{\prime}}\right)\right\}\right\|=\int \mathrm{d}^{6} z\left|\left(f_{z} \mid e^{-i \frac{\Delta}{2 m a}\left(t^{\prime}-t\right)} f_{z^{\prime}}\right)\right|=(2 \pi)^{3}\left(1+\left(\frac{t-t^{\prime}}{2 m_{a}}\right)^{2}\right)^{3 / 4}
$$

Explicitly the norm of the first order term of $\tau_{t}^{n}\left(a_{z^{\prime}}\right)$,

$$
\| \int_{0}^{t} \mathrm{~d} t_{1} \int_{\left|q_{1}\right|, p_{t} \mid \leq n} \mathrm{~d}^{6} z_{1} \mathrm{~d}^{6} z_{2} \mathrm{~d}^{6} z_{3} \int_{|q|=\text { const. }} \mathrm{d} O V(\ldots)\left[\begin{array}{l}
{\left[\tau _ { t _ { 1 } } ^ { \circ } \left(a_{z_{1}}^{*} a_{z_{1}}^{*} b_{z_{2}} b_{z_{2}} c_{z_{3}} c_{\hat{z}_{3}}\right.\right.} \\
\left.\left.+c_{z_{3}}^{*} c_{z_{3}}^{*} b_{z_{2}}^{*} b_{z_{2}}^{*} a_{z_{1}} a_{\hat{z}_{1}}\right), \tau_{t}^{\circ}\left(a_{z^{\prime}}\right)\right] \|
\end{array}\right.
$$

is bounded uniformly in $n$ by

$$
\begin{aligned}
& \leq \int_{0}^{t} \mathrm{~d} t_{1} \int \mathrm{d}^{6} z_{1} \mathrm{~d}^{6} z_{2} \mathrm{~d}^{6} z_{3} \int_{|q|=\text { const. }} d O\left|V\left(\left|q_{1}-q_{2}\right|,\left|q_{1}-q_{3}\right|,\left|\frac{p_{1}}{m_{a}}-\frac{p_{2}}{m_{b}}\right|,\left|\frac{p_{1}}{m_{a}}-\frac{p_{3}}{m_{c}}\right|, \vartheta_{q}, \vartheta_{p}\right)\right| \\
& \times\left\|\left[\tau_{t_{1}}^{\circ}\left(a_{z_{1}}^{*}\right) \tau_{t_{1}}^{\circ}\left(a_{z_{1}}^{*}\right), \tau_{t}^{\circ}\left(a_{z^{\prime}}\right)\right]\right\|\left\|\tau_{t_{1}}^{\circ}\left(b_{z_{2}}\right)\right\|\left\|\tau_{t_{1}}^{\circ}\left(b_{z_{2}}\right)\right\|\left\|\tau_{t_{1}}^{\circ}\left(c_{z_{3}}\right)\right\|\left\|\tau_{t_{1}}^{\circ}\left(c_{z_{3}}\right)\right\| \\
& \leq \int_{0}^{t} \mathrm{~d} t_{1} \int \mathrm{d}^{6} z_{1} \mathrm{~d}^{6} z_{2} \mathrm{~d}^{6} z_{3} \int_{|q|=\text { const. }} d O\left|V\left(\left|q_{1}-q_{2}\right|,\left|q_{1}-q_{3}\right|,\left|\frac{p_{1}}{m_{a}}-\frac{p_{2}}{m_{c}}\right|,\left|\frac{p_{1}}{m_{a}}-\frac{p_{3}}{m_{c}}\right|, \vartheta_{q}, \vartheta_{p}\right)\right| \\
& \times\left(\left\|\tau_{t_{1}}^{\circ}\left(a_{z_{1}}^{*}\right)\right\|\left\|\left\{\tau_{t_{1}}^{\circ}\left(a_{z_{1}}^{*}\right), \tau_{t}^{\circ}\left(a_{z^{\prime}}\right)\right\}\right\|+\left\|\left\{\tau_{t_{1}}^{\circ}\left(a_{z_{1}}^{*}\right), \tau_{t}^{\circ}\left(a_{z^{\prime}}\right)\right\}\right\|\left\|\tau_{t_{1}}^{\circ}\left(a_{z_{1}}^{*}\right)\right\|\right) \\
& \leq 2 \sup _{q_{1}, p_{1}} \int \mathrm{d}^{6} z_{2} \mathrm{~d}^{6} z_{3}|V(\ldots)| \times 4 \pi\left|\hat{q}^{2} \int \mathrm{d}^{6} z_{1} \int_{0}^{t} \mathrm{~d} t_{1}\right|\left(f_{z_{1}} \mid e^{-\imath \frac{\Delta}{2 m_{a}}\left(t-t_{1}\right)} f_{z^{\prime}}\right) \mid \\
& \leq 8 \pi|\hat{q}|^{2} m_{a}^{6}\|V\|_{1}|t|(2 \pi)^{3}\left(1+\left(\frac{t-t^{\prime}}{2 m_{a}^{2}}\right)^{2}\right)^{3 / 4} .
\end{aligned}
$$

Higher orders can be treated in a similar manner. We can assume that $m_{c}<m_{b}$, then

$$
\left\|\tau_{t}^{n}\left(a_{z^{\prime}}\right)\right\| \leq 1+2 \sum_{k=1}^{\infty} 10^{k}\left(4 \pi|\hat{q}|^{2} m_{a}^{6}\|V\|_{1}|t|(2 \pi)^{3}\left(1+\left(\frac{t-t^{\prime}}{2 m_{c}}\right)^{2}\right)^{3 / 4}\right)^{k}, \quad \forall z^{\prime} \in \mathbf{R}^{6}
$$

uniformly in $n \in \mathbf{N}$. By inspecting the perturbation formula (9) we establish the convergence of $\lim _{n \rightarrow \infty} \tau_{t}^{n}\left(a_{z^{\prime}}\right)$ for $|t|<t_{\text {。 }}$, with $t_{\text {。 }}$ given by

$$
2 \cdot(2 \pi)^{4}\left|\hat{q}^{2} m_{a}^{6}\|V\|_{1}\right| t_{0} \mid\left(1+\left(\frac{t_{\circ}}{2 m_{c}}\right)^{2}\right)^{3 / 4}=10^{-1}
$$


(iii) The coherent states $f_{z}$ form a total set in $L^{2}\left(\mathbf{R}^{3}\right)$, thus $\tau_{t}$ can be extended to arbitrary $a(f), f \in L^{2}\left(\mathbf{R}^{3}\right) . \quad \tau_{t}(b(f))$ and $\tau_{t}(c(f))$ are constructed in exactly the same manner and so $\lim _{n \rightarrow \infty} \tau_{t}^{n}(a), a \in \mathscr{A}$ exists for $t$ small enough by the Banach-Steinhaus theorem. By inspecting (9) one can see that in a neighbourhood of $t=0$ additivity in $\mathrm{t}$ holds for all $n \in \mathbf{N}$,

$$
\tau_{t 1}^{n} \circ \tau_{t 2}^{n}(a)=\tau_{t 1+t 2}^{n}(a), \quad a \in \mathscr{A}, \quad \forall t_{i} \in \mathbb{R}
$$

and since $\lim _{n \rightarrow \infty} \tau^{n}\left(a_{z^{\prime}}\right)$ converges for $|t|<t$ 。 we have,

$$
\tau_{t_{1}} \circ \tau_{t_{2}}\left(a_{z^{\prime}}\right)=\tau_{t_{1+t 2}}\left(a_{z^{\prime}}\right), \quad\left|t_{1}\right|+\left|t_{2}\right|<t_{\circ}
$$

This allows us to define $\tau_{t}$ for arbitrary $t \in \mathbf{R}$ :

$$
\tau_{t}\left(a_{z^{\prime}}\right):=\tau_{t_{1}}\left(\tau_{t 2}\left(\ldots\left(\tau_{t r}\left(a_{z^{\prime}}\right)\right) \ldots\right)\right) \quad t=t_{1}+t_{2}+\ldots+t_{r} \in \mathbb{R}, \quad\left|t_{l}\right|<t_{\circ}
$$

It even defines a ${ }^{*}$-endomorphism, as can be seen from the (anti-) commutator relations, e.g.,

$$
\left\{\tau_{t}(a(f)), \tau_{t}\left(a^{*}(g)\right)\right\}=\left\{a(f), a^{*}(g)\right\}
$$

Surjectivity is recovered from the group property: $\tau_{-t}$ provides an inverse for $\tau_{t}$, which shows surjectivity and consequently $\tau_{t}$ defines a ${ }^{*}$-isomorphism. Thus $\forall a \in \mathscr{A},\left\|\tau_{t}(a)\right\|=\|a\|$.

\section{§4. Galilei Invariance of the Time Evolution}

In nonrelativistic models we deal with nets of local observables $(I, \mathscr{O}) \rightarrow$ $(I, \mathscr{A}(\mathscr{O})), \quad I$ an open intervall, $\mathscr{O}$ an open region in $\mathbb{R}^{3}$, and $\mathscr{A}(\mathscr{O})$ the $C^{*}$-algebra corresponding to the respective region. We now equip $U(I, \mathscr{O}) \subset \mathbf{R} \times \mathbf{R}^{3}(I, \mathscr{A}(\mathscr{O}))^{C^{*}}$ with the bundle structure induced from the space-time structure (see Appendix). Recall that the kinematical automorphisms $\gamma^{\text {Kin }}$ have been specified in $\S 2$ by setting

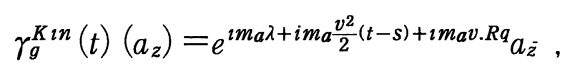

with $\bar{z}=\left(R q+v(t-s)+l, R p+m_{a} v\right)$.

Definition. Let $\hat{g}$ and $\hat{h}$ be elements of the isochronous Galilei group ${ }^{5}$

\footnotetext{
${ }^{5}$ For further details we refer the reader to the appendix.
} 
$G_{0}^{1}:=\left\{\left.(\lambda, s, l, v, R) \in G^{1}\right|_{s}=0\right\} . \quad$ Furthermore consider the transition functions

$$
\begin{aligned}
\Psi_{\hat{g}, \hat{h}:} \mathbf{R} & \rightarrow \quad \operatorname{Aut}(\mathscr{A}) \\
t & \rightarrow \gamma_{\hat{g}^{-1}}^{K i n}(\mathrm{t}) \circ \gamma_{\hat{h}}^{K i n}(t)
\end{aligned}
$$

We define the Galilean field algebra bundle to be the fiber bundle

$$
M_{\mathscr{A}}:=\left(\cup_{g \in G_{0}^{1}}\{\hat{g}\} \times \mathbf{R} \times \mathscr{A}\right) / \sim
$$

where the equivalence relation $\sim$ is given by $[\hat{g}, t, a] \sim\left[\hat{h}, t^{\prime}, a^{\prime}\right]: \Leftrightarrow t=t^{\prime}$ and $a^{\prime}=\Psi_{\hat{h}, \hat{g}}(t) a$.

The set of transition functions $\left\{\Psi_{\hat{g}, \hat{h}}\right\}_{\hat{g}, \hat{h} \in G_{0}^{1}}$ satisfies the necessary cocycle condition $\Psi_{\dot{g}, \hat{g}}=\gamma_{\hat{g}^{-1} \circ \hat{g}}^{K \imath n}=i d_{\mathscr{A}}$ and $\Psi_{\hat{g}, \hat{h}} \circ \Psi_{\hat{h} \hat{f}}=\gamma_{\hat{g}^{-1}}^{K \imath \circ} \gamma_{\hat{h}}^{K \imath n} \circ \gamma_{\hat{h}^{-1}}^{K \imath} \circ \gamma_{\hat{f}}^{K \imath n}=\gamma_{\hat{g}^{-1} \circ \hat{h} \circ \hat{h}^{-1} \circ \hat{f}}^{K \imath n}=\Psi_{\hat{g} \hat{f}, \text { so }}$ $\boldsymbol{M}_{\mathscr{A}}$ is well defined. Our intention is to show Galilei invariance of the time-evolution by defining it chart-independent on the Galilean field algebra bundle. We will use the following

Lemma 4.1. Let $g=(\lambda, s, l, v, R)$ and $g^{\prime}=\left(\lambda^{\prime}, s^{\prime}, l^{\prime}, v^{\prime}, R^{\prime}\right)$ denote two elements of $G^{1}$, The composition law of the automorphisms

$$
\gamma_{g}(t)(a):=\tau_{s} \circ \gamma_{(\lambda, 0, l, v, R)}^{K 2 n}(t)(a), \quad \forall a \in \mathscr{A}
$$

is given by

$$
\gamma_{g}\left(t+s^{\prime}\right) \circ \gamma_{g^{\prime}}(t)(a)=\gamma_{g \circ g^{\prime}}(t)(a), \quad \forall a \in \mathscr{A}
$$

where $t \in \mathbf{R}$.

It can be verifed by restricting $\gamma_{g}$ to one parameter subgroups, that Lemma 4.1 reproduces the relations used by Narnhofer and Thirring [6] to characterise Galilei invariance. We will see in a moment that this leads to a chart independent description.

Proof. Let denote $g=(0, \mathrm{~s}, 0,0, \mathbf{1}) \circ \hat{g}=(\hat{\lambda}, s, \hat{a}, \hat{v}, \hat{R})$. A straightforward computation yields

$$
\gamma_{g}\left(t+s^{\prime}\right) \circ \gamma_{g^{\prime}}(t)(a)=\tau_{s} \circ\left(\tau_{s^{\prime}}^{\circ} \circ \gamma_{g \circ g^{\prime}}^{K i n}(t)(a)+\right.
$$




$$
\begin{aligned}
\lim _{n \rightarrow \infty} \sum_{k=1}^{\infty} \frac{i}{k !} \int_{\left[0, s^{\prime}\right] k} \mathrm{~d}\left(t_{1} \ldots t_{k}\right)\left[\tau_{t_{k}}^{\circ} \circ \gamma_{\left(\lambda+\frac{1}{2} v t_{k}, 0, a+v t_{k}, v, R\right)}^{K i i_{1}}\left(t+s^{\prime}-t_{k}\right)\left(v_{n}\right),\right. \\
\left.\left.\quad\left[\ldots,\left[\tau_{t_{1}}^{\circ} \circ \gamma_{\left(\lambda+\frac{1}{2} v^{\left.2 i t_{1}, 0, a+v t_{1}, v, R\right)}\right.}^{i}\left(t+s^{\prime}-t_{1}\right)\left(v_{n}\right), \tau_{s^{\prime}}^{\circ} \circ \gamma_{\dot{g} \circ g^{\prime}}^{K i n}(t)(a)\right] \ldots\right]\right]\right)
\end{aligned}
$$

Thus it remains to show that the spatial symmetry gets restored, $\lim _{n \rightarrow \infty} \|\left[v_{n}-\right.$ $\left.\gamma_{\dot{g}}^{K \text { in }}(t)\left(v_{n}\right), a\right] \|=0$, for all $a \in \mathscr{A}$ and $t \in \mathbb{R}$. The following computation needs some care: one has to make sure that simple substitution of variables does not change the operator. This can be easily checked by computing $\left\{\gamma_{g}^{K \text { in }}(t)\left(v_{n}\right)\right.$, $\left.a_{z^{\prime}}\right\}$ with the different expressions on the right hand side of the following expression:

$$
\begin{aligned}
& \gamma_{g}^{K \text { in }}(t)\left(v_{n}\right)=\int_{\left|q_{1}\right|,\left|p_{1}\right| \leq n} \mathrm{~d}^{6} z_{1} \mathrm{~d}^{6} z_{2} \mathrm{~d}^{6} z_{3} \int_{|q|=\text { const. }} \mathrm{d} O V\left(\left|q_{1}-q_{2}\right|, \ldots, \vartheta_{p}\right) \\
& \times\left(e^{-2 v v R\left(m_{a} q_{1}-m_{b} q_{2}-m_{c} q_{3}\right)} a_{R q_{1}+v(t-s)+l, R p_{1}+m_{a} v}^{*} \ldots c_{R\left(q_{3}+\tilde{q}\right)+v(t-s)+l, R p_{3}+m_{a} v}\right.
\end{aligned}
$$

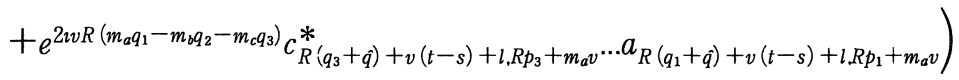

$$
\begin{aligned}
& =\int_{\substack{\left|R^{-1}\left(q_{1}-l-v t\right)\right| \leq n \\
\left|R^{-1}\left(p_{i}-m_{i} v\right)\right| \leq n}} \mathrm{~d}^{6} z_{1} \mathrm{~d}^{6} z_{2} \mathrm{~d}^{6} z_{3} \int_{\left|R^{-1} q\right|=c o n s t .} \mathrm{d} O V\left(\left|R^{-1}\left(q_{1}-l-v t\right)-R^{-1}\left(q_{2}-l-v t\right)\right|, \ldots, \vartheta_{p}\right) \\
& \times\left(a_{z_{1}}^{*} a_{\tilde{z}_{1}}^{*} b_{z_{2}} b_{\tilde{z}_{2}} c_{z_{3}} c_{\tilde{z}_{3}}+c_{z_{3}}^{*} c_{z_{3}}^{*} b_{z_{2}}^{*} b_{z_{3}}^{*} a_{z_{1}} a_{\hat{z}_{1}}\right) \\
& =\int \mathrm{d}^{6} z_{1} \mathrm{~d}^{6} z_{2} \mathrm{~d}^{6} z_{3} \int \mathrm{d} O V\left(\left|q_{1}-q_{2}\right|, \ldots, \vartheta_{p}\right)\left(a_{z_{1}}^{*} a_{z_{1}}^{*} b_{z_{2}} b_{\tilde{z}_{2}} c_{z_{3}} c_{z_{3}}+c_{z_{3}}^{*} c_{z_{3}}^{*} b_{z_{2}}^{*} b_{z_{3}}^{*} a_{z_{1}} a_{z_{1}}\right) . \\
& \left|q_{1}-a-v t\right| \leq n \quad|q|=\text { const. } \\
& \left|p_{1}-m_{1} v\right| \leq n
\end{aligned}
$$

The change of integration boundaries vanishes in the limit $n$ to infinity. Due to the strong continuity of $\tau^{\circ}$ this implies

$$
\begin{array}{r}
\mathrm{s}-\lim _{n \rightarrow \infty} \sum_{k=1}^{\infty} \frac{i^{k}}{k !} \int_{[0, s]^{k}} \mathrm{~d}\left(t_{1} \ldots t_{k}\right)\left[\tau_{t_{k}}^{\circ} \circ \gamma_{\dot{g}}^{K i n}\left(v_{n}\right),\left[\ldots,\left[\tau_{t_{1}}^{\circ} \circ \gamma_{\dot{g}}^{K \text { in }}\left(v_{n}\right), \tau_{s}^{\circ}(.)\right] \ldots\right]\right]= \\
\quad=\mathrm{s}-\lim _{n \rightarrow 0} \sum_{k=1}^{\infty} \frac{i^{k}}{k !} \int_{[0, s]^{k}} \mathrm{~d}\left(t_{1} \ldots t_{k}\right)\left[\tau_{t_{k}}^{\circ}\left(v_{n}\right),\left[\ldots,\left[\tau_{t_{1}}^{\circ}\left(v_{n}\right), \tau_{s}^{\circ}(.)\right] \ldots\right]\right],
\end{array}
$$

and consequently (34) holds.

Together with the kinematical automorphisms $\gamma^{\text {Kin }}$ the time evolution provides a representation of the extended Galilei group $G^{1}$ as bundle isomorphisms on $\boldsymbol{M}_{\mathscr{A}}$ : 
Theorem 4.2. Let $\hat{h}=(\hat{\lambda}, 0, \hat{l}, \hat{v}, \hat{R}) \in G_{0}^{1}$ and $g=(\lambda, s, l, v, R) \in G^{1}$. Then II: $G^{1} \rightarrow I s o\left(M_{\mathscr{A}}\right)$, with

$$
\begin{aligned}
& g \quad \rightarrow \quad i_{g}: \quad M_{\mathscr{A}} \rightarrow M_{\mathscr{A}} \\
& {[\hat{h}, t, a] \rightarrow\left[\hat{h}^{\prime}, t+s, \gamma_{g}(t)(a)\right]}
\end{aligned}
$$

with $\hat{h}^{\prime}=(\hat{\lambda}, s, \hat{l}, \hat{v}, \hat{R}) \circ g^{-1}$, defines a representation of $G^{1}$ as fiber bundle-isomorphisms on $M_{\mathscr{A}}$.

Proof. (i) First we show that $i_{g}$ does not depend on the representant of the equivalence class: Let $[\hat{g}, t, a] \sim\left[\hat{g^{\prime}}, t^{\prime}, a^{\prime}\right]$ be two representants of the same equivalence class. Then (by definition) $t=t^{\prime}$ and $a^{\prime}=\Psi_{\hat{h}^{\prime}, \hat{h}}(t) a$, therefore $t+s=$ $t^{\prime}+s$. Furthermore

$$
\begin{aligned}
\gamma_{g}(t)\left(a^{\prime}\right) & =\gamma_{g}(t)\left(\Psi_{\hat{h}^{\prime}, \hat{h}}(t)(a)\right) \\
& =\left(\gamma_{\left(\hat{h}^{\prime} \circ g^{-1}\right)^{-1} \circ \gamma_{h^{\circ} \circ g^{-1}}}\right)(t+s) \circ \gamma_{g}(t)(a) \\
& =\Psi_{h, h^{\prime}}(t+s)\left(\gamma_{g}(t)(a)\right),
\end{aligned}
$$

with $\check{h}=\left(\hat{\lambda}^{\prime}, s, \hat{l}^{\prime}, \hat{v}^{\prime}, \hat{R}^{\prime}\right) \circ g^{-1}$ and $\check{h}^{\prime}=\left(\hat{\lambda}^{\prime}, s, \hat{l}^{\prime}, \hat{v}^{\prime}, \hat{R}^{\prime}\right) \circ g^{-1}$. From (39) and the definition (38) we infer $i_{g}([\hat{g}, t, a]) \sim i_{g}\left(\left[\hat{g}^{\prime}, t^{\prime}, a^{\prime}\right]\right)$, and thus $i_{g}$ is well defined. (ii) The group multiplication law holds:

$$
\begin{aligned}
i_{g} \circ i_{g^{\prime}}([\hat{h}, t, a]) & \left.=i_{g}\left(\left[\hat{\lambda}, s^{\prime}, \hat{l}, \hat{v}, \hat{R}\right) \circ g^{\prime-1}, t+s^{\prime}, \gamma_{g^{\prime}}(t)(a)\right]\right) \\
& =\left[\left(\hat{\lambda}, s+s^{\prime}, \hat{l}, \hat{v}, \hat{R}\right) \circ\left(g \circ g^{\prime}\right)^{-1}, t+\left(s+s^{\prime}\right), \gamma_{g \circ g^{\prime}}(t)(a)\right] \\
& =i_{g \circ g^{\prime}}([\hat{h}, t, a])
\end{aligned}
$$

for all $g, g^{\prime} \in G^{1}$.

Corollary 4.3. $\tau_{s}$ defines a Galilei invariant time evolution.

Proof. The restriction of $\Pi$ to the time component, $\Pi_{\mid \text {time }}: \mathbf{R} \rightarrow I s o\left(\mathcal{M}_{\mathscr{A}}\right)$ :

$$
\begin{aligned}
& s \rightarrow i_{s}: \quad M_{\mathscr{A}} \quad \rightarrow \quad M_{\mathscr{A}} \\
& {[\hat{h}, t, a] \rightarrow\left[\hat{h}, t+s, \tau_{s}(a)\right]}
\end{aligned}
$$

defines the time evolution independent of the chart. 


\section{§5. Equilibrium and Ground states}

True (pure phase) equilibrium states are distinguished by strict invariance under time translations and stability against local perturbations. If we add some technical assumptions, then these requirements are equivalent [28-30] to a characterising property first found by Kubo, Martin and Schwinger [31-32]: A state $\omega$ over $\mathscr{A}$ is defined to be a $(\tau, \beta)-K M S$ state, if

$$
\omega(a b)=\omega\left(b \tau_{i \beta}(a)\right)
$$

for all $a, b$ in a norm dense, $\tau$-invariant ${ }^{*}$-subalgebra of $\mathscr{A}_{\tau}$, where $\mathscr{A}_{\tau}$ denotes the set of analytic elements for $\tau$. The GNS-representation $\left(\mathscr{H}_{\omega}, \pi_{\omega}, \Omega_{\omega}\right)$ associated with an equilibrium state $\omega_{\beta}$ connects our state-independent results with the more common equilibrium formalism. While the laws of physics have to be Galilei invariant, the KMS condition distinguishes a rest frame [33]. The breakdown of Galilei invariance is an unavoidable consequence of the Tomita-Takesaki theory: Any symmetry not commuting with time translations can not leave a KMS state (over a simple $C^{*}$-algebra) invariant ([34], see also $[35,36])$. If we want to describe a KMS state from a different inertial frame, we have to use the following covariant KMS condition,

$$
\omega(a b)=\omega\left(b_{\kappa_{1 \beta}}(a)\right)
$$

where $\left\{\kappa_{t}\right\}_{t \in \mathbf{R}} \subset\left\{\gamma_{g}\right\}_{g \in G^{1}}$ denotes the one parameter group of automorphism corresponding to the central element $\mathbb{U}=\mathbf{H}-\mathbb{P}^{2} / 2 m$ of the extended Galilei group (see Appendix).

Once we have established our $C^{*}$-dynamical system $(\mathbb{R}, \mathscr{A}, \tau)$, the existence of equilibrium states for our model follows from the literature. We shortly outline the line of results. The free time evolution respects the tensor product structure of $\mathscr{A}$,

$$
\tau^{\circ}\left(a \otimes b \otimes_{c}\right)=\tau^{\circ}(a \otimes \mathbb{1} \otimes \mathbb{1}) \otimes \tau^{\circ}(\mathbb{1} \otimes b \otimes \mathbb{1}) \otimes \tau^{\circ}\left(\mathbf{1} \otimes \mathbb{1} \otimes_{c}\right),
$$

for all $a \in \mathscr{A}_{a}$, etc. The unique $\left(\tau^{\circ}, \beta, \mu_{a}, \mu_{b}, \mu_{c}\right)$-KMS state [8], where $\beta=$ $1 / k T$ is the inverse temperature and $\mu_{a}, \mu_{b}, \mu_{c}$ are the chemical potentials for the different species, is given by

$$
\omega_{\beta}^{\circ}\left(a \otimes b \otimes_{c}\right)=\omega_{\beta, \mu a}^{\circ}(a) \omega_{\beta, \mu b}^{\circ}(b) \omega_{\beta, \mu c}^{\circ}(c)
$$

for all $a \in \mathscr{A}_{a}$, etc., with 


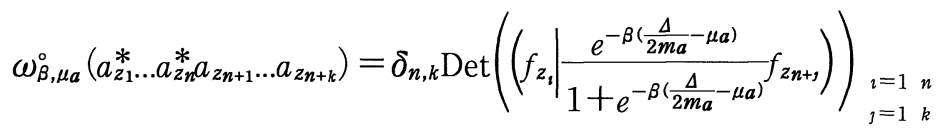

and simlar for $b$ and $c$. In order to see how the three chemical potentials enter one has to examine the role of the $U(1)$ gauge invariance more closely $[37,38]$.

Even if we allow chemical reactions only in a local region, the situation changes dramatically: The tensor product structure is destroyed and we lose control over the relative particles densities. The chemical reaction itself will abjust the equilibrium relative densities, depending on the temperature. Nevertheless, for each initial choice of $\left(\beta, \mu_{a}, \mu_{b}, \mu_{c}\right)$ and each $n \in \mathbf{N}$ there exists ([8], Prop. 5.4.1 and Cor. 5.4.5) exactly one $(\tau, \beta)$-KMS state $\omega_{\beta}^{n}$, when we switch on the interaction ${ }^{6}$. The existence of KMS states in the limit $n \rightarrow \infty$ follows from standard arguments: the unit ball in $\mathscr{A}_{F}{ }^{*}$ is weak ${ }^{*}$ compact, thus there exists a subsequence ${ }^{7}$ of $\left\{\omega_{\beta}^{n}\right\}_{n \in N}$ converging to some state $\omega_{\beta}$. It remains to show that $\omega_{\beta}$ satisfies the KMS relation. A priory one can not be sure that the intersection of the sets of analytic elements for the different automorphisms is dense, but this problem can be avoided by using a different formulation of the KMS condition ([8], 5.3.12) emphasising the properties of analyticity: Let $\mathscr{D}$ denote the set of infinitely differentiable functions with compact support in $\mathbf{R}$. If $\widetilde{f} \in \mathscr{D}$, then the inverse Fourier transform $f$ is an entire analytic function and since $\lim _{n \rightarrow \infty} \tau^{n}$ exists with respect to the strong topology on the automorphism group,

$$
\begin{gathered}
\int_{-\infty}^{\infty} \mathrm{d} t f(t) \omega_{\beta}\left(a \tau_{t}(b)\right)=\lim _{n \rightarrow \infty} \int_{-\infty}^{\infty} \mathrm{d} t f(t) \omega_{\beta}^{n}\left(a \tau_{t}^{n}(b)\right)=\lim _{n \rightarrow \infty} \int_{-\infty}^{\infty} \mathrm{d} t f(t+i \beta) \omega_{\beta}^{n}\left(\tau_{t}^{n}(b) a\right) \\
=\int_{-\infty}^{\infty} \mathrm{d} t f(t+i \beta) \omega_{\beta}\left(\tau_{t}(b) a\right)=\int_{-\infty}^{\infty} \mathrm{d} t f(t) \omega_{\beta}\left(\tau_{t-\imath \beta}(b) a\right) \quad \forall a, b \in \mathscr{A}_{\tau} .
\end{gathered}
$$

Now choose $\widetilde{f_{k}} \in \mathscr{D}$ such that $0 \leq \widetilde{f}_{k} \leq 1, \widetilde{f}_{k}(x)=1$ if $|x| \leq k$ and $\widetilde{f_{k}}(x)=0$ if $|x| \geq k$ +1 . Since $\widetilde{f}_{k} \stackrel{w *}{\rightarrow} 1 \in \mathscr{D}^{\prime}$, it follows that $f_{k} \stackrel{w *}{\rightarrow} \delta$. Thus, for any bounded, continuous function $g$,

$$
\lim _{k \rightarrow \infty} \int_{-\infty}^{\infty} \mathrm{d} t f_{k}(t) g(t)=g(0)
$$

\footnotetext{
${ }^{6}$ The reverse is of course false. Different initial states can lead to the same interacting KMS state.

${ }^{7}$ Since $\mathscr{A}_{F}$ is separable the weak ${ }^{*}$ topology is metrizable on bounded subsets of $\mathscr{A}_{F}{ }^{*}$.
} 
and hence $\omega_{\beta}(a b)=\omega_{\beta}\left(\tau_{-i \beta}(b) a\right)$ for all $a, b \in \mathscr{A}_{\tau}$. Uniqueness in the thermodynamic limit $n \rightarrow \infty$ can no longer be expected for all temperatures, since phase transitions might occur. There is no uniform convergence $\lim _{n \rightarrow \infty} \sup _{\|\boldsymbol{a}\|=1}\left\|\tau_{t}^{n}(a)-\tau_{t}(a)\right\|=0$. Consequently also the representations given by the KMS state $\omega_{\beta}^{\circ}$ and $\omega_{\beta}$ will not be quasi-equivalent, which means that there will be no weakly continuous isomorphism between the corresponding von Neumann algebras. $\omega_{\beta}^{\circ}$ is space translation-invariant and the extremal translation-invariant components of $\omega_{\beta}$ will not admit another normal translation-invariant state [6].

\section{\$6. Appendix}

In non-relativistic classical as well as quantum physics it is a basic assumption that the laws of nature have a Galilei invariant meaning i.e., they are independent of the actual time, the position, the orientation and the center of mass momentum of the described physical system. Two descriptions of a series of physical events are equivalent if they can be connected by a coordinate transformation induced by an element $g=(s, l, v, R) \in \mathbf{R} \otimes \mathbb{R}^{3} \otimes \mathbb{R}^{3} \otimes O(3, \mathbf{R})$ of the proper Galilei group G:

$$
x^{\prime}=R x+v t+l, \quad t^{\prime}=t+s
$$

Starting from an arbitrary coordinate system we can label equivalent frames by the corresponding group element $g \in G$. Space-time, or more precisely, the space-time manifold $M_{G}$ is constructed by gluing different frames together and then identifying equivalent points in different charts. While in Aristotelian physics both, space and time, were absolute, every event defined by an instant of time and a location in space, in Galilean physics space becomes relative: the space-like distance of two space-time points with different time components has no a priori meaning. Time remains absolute and gives rise to a (fiber-bundle) projection $p_{G}: M_{G} \rightarrow \mathbb{R}$, providing a universal synchronisation prescription for watches $[39,40]$. Once the watches are synchronised in different charts, the group connecting equivalent charts is reduced to the isochronous Galilei group $G_{0}:=\left\{\left.(s, l, v, R) \in G\right|_{s}=0\right\}$. This is the starting-point for our construction of the space-time fiber bundle. Equivalent points in the charts $\tilde{g}$ and $\tilde{h} \in G_{0}$ are identified by transition functions $\phi_{\tilde{g}, \tilde{h}}(t)$ acting in the fiber $\mathbf{R}^{3}$ (= space) and depending on the base point $t \in \mathbb{R}$ (=time).

Definition. Let $\tilde{g}, \tilde{h} \in G$ 。 and $E_{3}$ denote the Euclidean group in three dimensions. Furthermore, consider the following transition functions 


$$
\begin{array}{rlc}
\phi_{\tilde{g} \tilde{h}:} \quad \mathbf{R} & \rightarrow & E_{3} \\
t & \rightarrow e_{\bar{g}}(t)^{-1} \mathrm{O}_{e \bar{h}}(t)
\end{array}
$$

where $e_{g}(t) x:=R x+v t+l$. The space-time manifold $M_{G}$,

$$
M_{G}:=\left(U_{\tilde{g} \in G_{0}}\{\tilde{g}\} \times \mathbf{R} \times \mathbf{R}^{3}\right) / \sim,
$$

is described by the equivalence relations $[\tilde{g}, t, x] \sim\left[\tilde{h}, t^{\prime}, x^{\prime}\right]: \Leftrightarrow t=t^{\prime}$ and $x^{\prime}=$ $\phi_{\tilde{h} \tilde{g}}(t) x$. The bundle projection is given by $p_{G}([\tilde{g}, t, x])=t$. Galilean space-time is defined to be the fiberbundle $\left(\mu_{G}, p_{G}, \mathbf{R}\right)$ with fiber $\mathbb{R}^{3}$.

Although $M_{G}$ is split up into space and time components in every (uniformly moving) coordinate system $\tilde{g}: M_{G} \rightarrow \mathbf{R} \times \mathbf{R}^{3}$, there is no canonical projection from $M_{G} \rightarrow \mathbb{R}^{3} . \quad M_{G}$ is trivializeable, but not canonically trivial. By definition, the set $\left\{\phi_{\tilde{g}, \tilde{h}}\right\}_{\tilde{g}, \tilde{h} \in G_{o}}$ fulfills the cocycle conditions $\phi_{\tilde{g} \tilde{h}} \circ \phi_{\tilde{h} \tilde{f}}=\phi_{\tilde{g} \tilde{f} \tilde{f}}$ and $\phi_{\bar{g}, \tilde{g}}=i d$ on $\mathbb{R}^{3}$, the required compatibility condition for the construction of fiberbundles [41].

The Galilei group was analysed in detail by Lévy-Leblond [20]. We collect a few facts relevant for a discussion of the present model. Each element of the Galilei group $G$ can be written as a product of a time translation $(s, 0,0,1)$, a space translation $(0, l, 0,1)$, a pure Galilei transformation $(0,0, v, \mathbf{1})$ and a rotation $\left(0,0,0, R_{\theta}\right)$ with generators $\mathbf{H}, \mathbf{P}, \mathbf{X}, \mathbf{J}$ respectively:

$$
\begin{aligned}
\left(s, a, v R_{\theta}\right) & =(s, 0,0, \mathbf{1}) \circ(0, a, 0, \mathbf{1}) \circ(0,0, v, \mathbf{1}) \circ\left(0,0,0, R_{\theta}\right) \\
& =e^{-\imath \mathbf{H} s} e^{-\imath \mathbf{P} l} e^{-\imath \mathbf{X} m v} e^{-\imath \mathbf{J} \theta},
\end{aligned}
$$

where $\theta$ denotes the three rotation angles. A scaling factor $m \in \mathbf{R}^{+}$was introduced in front of $v$, so that the notation is closer to the standard notation in classical mechanics. So far space and momentun translations commute, as can be seen from the group multiplication law. In classical mechanics the observables form an abelian algebra of functions over phase space $T^{*}\left(\mathbf{R}^{3 N}\right)$. The generators $\mathbf{P}, \mathbf{X}, \mathbf{J}$ and $\mathbf{H}$ are identified with the momentum, position, angular momentum and energy of the physical system.

In quantum theory this scheme is generalised by releasing the commutativity of space and time translations. Since Galilei invariance should not be affected by gauge transformations, a central extension of the Galilei group seems appropriate. $U(1)$, the gauge group of classical electromagnetism, is the minimal choice.

Definition. Let $m \in \mathbb{R}$. The extended Galilei group $\left(G^{m}, \circ\right)$ is the eleven 
parameter group $g=(\lambda, s, a, v, R) \in S^{1} \times \mathbf{R} \times \mathbf{R}^{3} \times \mathbf{R}^{3} \times O(3, \mathbb{R})$ with the group multiplication $\circ$ defined by ${ }^{8}$

$$
\begin{aligned}
& (\lambda, s, l v, R) \circ\left(\lambda^{\prime} s^{\prime}, l^{\prime} v^{\prime}, R^{\prime}\right)= \\
& \left(\lambda+\lambda^{\prime}+m\left(\frac{v^{2} s^{\prime}}{2}+v \cdot R l^{\prime}\right), s+s^{\prime}, l+R l^{\prime}+v s^{\prime}, v+R v^{\prime}, R R^{\prime}\right) .
\end{aligned}
$$

Let $\mathbf{M}$ denote the generator of the gauge transformations. Then the Lie algebra of the extended Galilei group $G^{m}$ is characterised by the relations:

$$
\begin{array}{lll}
{\left[\mathbf{J}_{\imath}, \mathbf{J}_{j}\right]=i \epsilon_{i j k} \mathbf{J}_{k}} & {\left[\mathbf{J}_{i}, \mathbf{H}\right]=0} & {\left[\mathbb{P}_{\imath}, \mathbb{P}_{j}\right]=0} \\
{\left[\mathbf{J}_{i}, \mathbf{X}_{j}\right]=i \epsilon_{i j k} \mathbf{X}_{k}} & {\left[\mathbb{J}_{i}, \mathbf{M}\right]=0} & {\left[\mathbb{P}_{i}, \mathbf{H}\right]=0} \\
{\left[\mathbf{J}_{i}, \mathbf{P}_{j}\right]=i \epsilon_{i j k} \mathbf{P}_{k}} & {\left[\mathbf{X}_{i}, \mathbf{X}_{j}\right]=0} & {\left[\mathbf{P}_{i}, \mathbf{M}\right]=0} \\
{\left[\mathbf{X}_{i}, \mathbf{P}_{j}\right]=i \delta_{i j}} & {\left[\mathbf{X}_{\imath}, \mathbf{M}\right]=0} & {[\mathbf{H}, \mathbf{M}]=0} \\
{\left[m \mathbf{X}_{i}, \mathbf{H}\right]=i \mathbf{P}_{i}} & &
\end{array}
$$

As argued, the extension is central, so $\mathbf{M}$ commutes with all other Lie algebra elements. The total mass $\mathbf{M}$, the internal energy $\mathbf{U}:=\mathbb{H}-\mathbf{P}^{2} / 2 m$ and the spin, i.e., the internal angular momentum $\mathbf{S}^{2}:=(\mathbb{J}-\mathbf{X} \times \mathbf{P})^{2}=s(s+1)$ generate a three dimensional center of the group and are therefore chart independent properties associated with a particle.

As pointed out in [19], the difference between Galilei and Poincare invariance is that, if we add an interaction $\mathbf{V}$ to the Hamiltonian $\mathbf{H}=\mathbf{H}_{\circ}+\mathbf{V}$, the commutation relations are not modified in the Galilean case, provided that $\mathbb{V}$ commutes with $(\mathbb{P}, \mathbb{J}, \mathbf{K}, \mathbf{M})$, while in the relativistic case any modification of the Hamiltonian requires a subsequent modification of other elements of the Lie algebra, since the Hamiltonian appears in the commutator of the generators of space translations and pure Lorentz transformations.

All the quantities usually considered as physically relevant, namely the energy, momentum, position, angular momentum and mass, appear as generators of the (extended) Galilei group. The problem is that for systems with an infinite number of particles quantities like the Hamiltonian or even the particle number are not well defined without reference to a Hibert space representation. Here we present a representation of the Lie algebra of $G^{m}$ as an algebra of derivations acting on $\mathscr{A}$.

Proposition 6.1. Each $\gamma_{g}(t), g \in G^{m}$ can be written as a product of derivable automorphisms,

\footnotetext{
${ }^{8}$ Here $m \in \mathbf{R}$ denotes a parameter labeling different extensions of the Galilei group. Note that the factor $m_{a}$ (resp. $m$ ) in (4) (resp. (5)) has a somewhat different origin.
} 


$$
\gamma_{g}(t)=\gamma_{(\lambda, 0,0,0,1)} \circ \gamma_{(0, s, 0,0,1)} \circ \gamma_{(0,0,1,0,1)} \circ \gamma_{(0,0,0, v, 1)}(t) \circ \gamma_{\left(0,0,0,0, R_{\theta}\right)}
$$

The corresponding derivations $\delta_{\mathbf{M}}, \delta_{\mathbf{H}}, \delta_{\mathbf{P}}, \delta_{\mathbf{X}}$ and $\delta_{\mathbf{J}}$ define a representation of the Lie algebra of $G^{m}$ on a common dense set $\mathscr{D}$,

$$
\begin{aligned}
& {\left[\delta_{\mathbf{J}_{i},}, \delta_{\mathbf{J}_{i}}\right]=i \epsilon_{\imath j k}, \delta_{\mathbf{J}_{k}} \quad\left[\delta_{\mathbf{J}_{i}}, \delta_{\mathbf{H}}\right]=0 \quad\left[\delta_{\mathbf{P}_{i},}, \delta_{\mathbf{P}}\right]=0}
\end{aligned}
$$

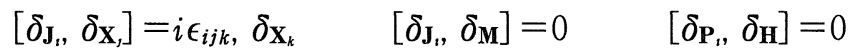

$$
\begin{aligned}
& {\left[\delta_{\mathbf{J}_{i}}, \delta_{\mathbf{P}_{t}}\right]=i \epsilon_{i j k}, \delta_{\mathbf{P}_{k}} \quad\left[\delta_{\mathbf{x}_{i}}, \delta_{\mathbf{X}_{\jmath}}\right]=0 \quad\left[\delta_{\mathbf{P}_{i}}, \delta_{\mathbf{M}}\right]=0}
\end{aligned}
$$

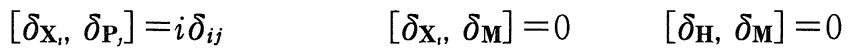

$$
\begin{aligned}
& {\left[\delta_{\mathbf{X}_{i}}, \delta_{\mathbf{H}}\right]=\frac{i}{m} \delta_{\mathbf{P}_{1}}}
\end{aligned}
$$

where the Lie-bracket is defined by $\left[\delta, \delta^{\prime}\right](a):=\delta\left(\delta^{\prime}(a)\right)-\delta^{\prime}(\delta(a)), \forall a \in \mathscr{A}$. (Note that $\delta_{i j}$ is the Kronecker symbol, while all the other $\delta$ 's denote derivations.)

Proof. Let $\mathscr{D}=\left\{b_{n} \mid b \in \mathscr{A}\right\}$, with

$$
b_{n}:=\left(\frac{n}{\pi}\right)^{7 / 2} \int \gamma_{(0, s, l, v, 1)}(b) e^{-n\left(s^{2}+l^{2}+v^{2}\right)} \mathrm{d} s \mathrm{~d}^{3} l \mathrm{~d}^{3} v, \quad b \in \mathscr{A}
$$

$\left\|b_{n}\right\| \leq\|b\|$ for all $n$, and $b_{n} \rightarrow b$ in the norm topology as $n \rightarrow \infty$. It follows ([7], 2.5.22.) that each $b_{n}$ is an analytic element for $\gamma_{g}$. For example, there exists a derivation $\delta \mathbf{P}$,

$$
\left(\delta_{\mathbf{P}}\right)^{k}\left(b_{n}\right)=\lim _{m \rightarrow \infty}\left[\int_{|q|,|p| \leq m} \mathrm{~d}^{6} z p a_{z}^{*} a_{z}+\int_{|q|,|p| \leq m} \mathrm{~d}^{6} z p b_{z}^{*} b_{z}+\int_{|q|,|p| \leq m} \mathrm{~d}^{6} z p c_{z}^{*} c_{z}, b_{n}\right],
$$

$b_{n} \in \mathscr{D}$, such that

$\gamma(0,0 . l, 0,1)\left(b_{n}\right)=\sum_{k>0} \frac{\left(l \delta \mathbf{S}_{\mathbf{P}}\right)^{k}\left(b_{n}\right)}{k !}$ and $\sum_{k>0} \frac{|l|^{k}}{k !}\left\|\delta_{\mathbf{P}}^{k}\left(b_{n}\right)\right\|<+\infty, \quad \forall l \in U \subset \mathbf{R}^{3}$,

for some open neighbourhood $U$ of the origin in $\mathbf{R}^{3}$. The multiplication table (56) can be established by direct computation.

\section{Acknowledgments}

The authors want to thank Martin Neuwirther for discussion of the classical Galilei bundle and C. J. wants to thank D. Buchholz for valuable suggestions on several points for the present version of the manuscript. 


\section{References}

[1] Requardt, M., Spectrum Condition, Analyticity, Reeh-Schlieder and Cluster Properties in Non-relativistic Galilei Invariant Quantum Theory, J. Phys. A: Math. Gen., 15 (1982), 37153723.

[2] Lévy-Leblond, J. M., Galilean Quantum Field Theory and a Ghostless Lee Model, Comm. Math. Phys., 4 (1967), 157-176.

[3] Schrader, R., On the Existence of a Local Hamiltonian in the Galilean Invariant Lee Model. Comm. Math. Phys., 10 (1968), 155-178.

[4] Hagen, C. R., Galilean Invariant Lee Model for All Spins and Parities Comm. Math. Phys., 21 (1971), 219-236.

[5] Narnhofer, H. and Thirring, W., Quantum Field Theories with Galilei-Invariant Interactions, Phys. Rev. Lett., 64 (1990), 1863-1866.

[6] - Galilei-Invariant Quantum Field Theories with Pair Interactions, Int. J. Mod. Phys., A6 (1991), 2937-2970.

[7] Bratteli, O. and Robinson. D. W., Operator Algebras and Quantum Statistical Mechanics. I, Springer-Verlag, New York-Heidelberg-Berlin, 1979

[8] - Operator Algebras and Quantum Statistical Mechanics. II, Springer-Verlag, New York-Heidelberg-Berlin, 1981.

[9] Emch, G. G., Algebraic Methods in Statistical Mechanics and Quantum Field Theory, Wiley, New York, 1972.

[10] Thirring, W., A Course in Mathematical Physics IV, Springer-Verlag, Berlin-Heidelberg-New York, 1983.

[11] Narnhofer, H. and Thirring W.. Mixing Properties of Quantum Systems. J. Stat. Phys., 57 (1989), 811-825.

[12] Narnhofer H., Pflug, A. and Thirring W., Mixing and Entropy Increase in Quantum Systems, Estatto da "Symmetry in Nature" Volume in honour of Luigi A. Radicati di Brozolo. Scuola Normale Superiore, (1989). Pisa.

[13] Narnhofer H., Thirring, W. and Wiklicky H., Transitivity and Ergodicity of Quantum Systems, J. Stat. Phys., 52 (1988), 1097-1112.

[14] Narnhofer, H. and Thirring, W., Ergodic Properties in Quantum Field Theories, Beer-Sheva Proceedings, to appear.

[15] Benatti, F., Deterministic chaos in infinite quantum systems. Springer Verlag, Berlin, 1993.

[16] Ohya, M. and Petz, D., Quantum Entropy and its Use, Springer-Verlag Berlin Heidelberg, 1993.

[17] Jäkel, C. D., Asymptotic Triviality of the Møller Operators in Galilei Invariant Quantum Field Theories, Lett. Math. Phys., 21 (1991), 343-350.

[18] Powers, R. T. and Sakai, S., Existence of Ground States and KMS States for Approximately Inner Dynamics, Comm. Math. Phys., 39 (1975), 273-288.

[19] Bargmann, V., On Unitary Ray Representations of Continuous Groups, Ann. Math., 59 (1952). $1-46$.

[20] Lévy-Leblond, J. M., Galilei Group and Galilei Invariance, in Loebl: Group Theory II. Academic Press, New York, (1971).

[21] Marmo, G. and Whiston, G. S., The Group of Automorphisms of the Galilei Group, Int. J. Theo. Phys., 6 (1972), 293-299.

[22] Havas, P. and Plebanski, J., Conformal Extensions of the Galilei Group and their Relations to the Schrödinger Group, J. Math. Phys., 19 (1978), 482-488. 
[23] Elizalde, E., Poincaré is a Subgroup of Galilei in One Space Dimension More, J. Math. Phys., 19 (1978), 526-528.

[24] Cattaneo, U., The Quantum Mechanical Poincaré and Galilei Group. J. Math. Phys., 19 (1978), 767-773.

[25] Brooke, J. A., A Galileian Formulation of Spin I. Clifford Algebras and Spin Groups, J. Math. Phys., 19 (1978), 952-959.

[26] Cassinelli, G. and Lahti, P. J.. Nontransitive imprimitivity systems for the Galilei group, $J$. Math. Phys., 31 (1990), 1859-1861.

[27] Guenin, M., On the Interaction Picture, Comm. Math. Phys., 3 (1966), 120-132.

[28] Haag, R., Hugenholtz, N. M. and Winnink, M., On the equilibrium states in quantum statistical mechanics, Comm. Math. Phys., 5 (1967), 215-236.

[29] Narnhofer, H. and Thirring W., Adiabatic Theorem in Quantum Statistical Mechanics, Phys. Rev. A., 26 (1982), 3646-3652.

[30] Haag, R., Kastler, D. and Trych-Pohlmeyer, E. B., Stability and Equilibrium States, Comm. Math. Phys., 38 (1974), 173-193.

[31] Kubo, R., Statistical mechanical theory of irreversible processes I., J. Math. Soc. Japan., 12 (1957), 570-586.

[32] Martin, P. C. and Schwinger, J., Theory of Many-Particle Systems. I, Phys. Rev., 115 (1959), 1342-1373.

[33] Swieca, J. A., Range of Forces and Broken Symmetries in Many-Body Systems, Comm. Math. Phys., 4 (1967), 1-7.

[34] Herman, R. H. and Takesaki, M., States and automorphism groups of operator algebras. Comm. Math. Phys., 19 (1970), 142-160.

[35] Narnhofer, H., Kommutative Automorphismen und Gleichgewichtszustände, Act. Phys. Aust., 47 (1977), 1-29.

[36] Ojima, I., Lorentz Invariance vs. Temperature in QFT, Lett. Math. Phys., 11 (1986), 73-80.

[37] Araki, H., Haag, R., Kastler, D. and Takesaki, M., Extension of KMS States and Chemical Potential, Comm. Math. Phys., 53 (1977), 97-134.

[38] Araki, H. and Kishimoto, A., Symmetry and Equilibrium States, Comm. Math. Phys., 52 (1977), 211-232.

[39] Trautman, A., Fibre bundles associated with space-time, Rep. Math. Phys., 1 (1970), 29-62.

[40] Thirring, W., A Course in Mathematical Physics I, Springer-Verlag, Berlin-Heidelberg-New York, 1978.

[41] Dieudonné, J., Foundations of Modern Analysis III, Academic Press, New York, 1972. 
